\title{
Surgical Safety in Pediatrics: practical application of the Pediatric Surgical Safety Checklist ${ }^{1}$
}

\author{
Maria Paula de Oliveira Pires ${ }^{2}$ \\ Mavilde L. G. Pedreira ${ }^{3}$ \\ Maria Angélica Sorgini Peterlini ${ }^{3}$
}

\begin{abstract}
Objectives: to assess the practical application of the Pediatric Surgical Safety Checklist on the preoperative period and to verify family satisfaction regarding the use of the material. Method: exploratory study that aimed to analyze the use of the checklist by children who underwent surgical interventions. The sample was constituted by 60 children (from preschoolers to teens) and 60 family members. The variables related to demographic characterization, filling out the checklist, and family satisfaction, being evaluated through inferential and descriptive statistical analysis. Results: most children (71.7\%) were male, with a median age of 7.5 years. We identified the achievement of $65.3 \%$ of the checklist items, $30.0 \%$ were not filled due to non-performance of the team and $4.7 \%$ for children and family reasons. In the association analysis, we found that the removal of accessories item ( $p=0.008$ ) was the most checked by older children. Regarding satisfaction, the family members evaluated the material as great (63.3\%) and good (36.7\%) and believed that there was a reduction of the child's anxiety (83.3\%). Conclusion: the use of the checklist in clinical practice can change health services regarding safety culture and promote customer satisfaction.
\end{abstract}

Descriptors: Patient Safety; Pediatric Nursing; Preoperative Care.

\footnotetext{
1 Paper extracted from master's thesis "Cirurgia Segura em Pediatria: Elaboração e Validação de Checklist de Intervenções Pré-operatórias", presented to Escola Paulista de Enfermagem, Universidade Federal de São Paulo, São Paulo, SP, Brazil. Supported by Conselho Nacional de Desenvolvimento Científico e Tecnológico (CNPq), Brazil, process \# 476088/2010-0.

2 Doctoral student, Escola Paulista de Enfermagem, Universidade Federal de São Paulo, São Paulo, SP, Brazil. Assistant Professor, Instituto de Ciências da Saúde, Universidade Paulista, São Paulo, SP, Brazil.

3 PhD, Associate Professor, Escola Paulista de Enfermagem, Universidade Federal de São Paulo, São Paulo, SP, Brazil.
}

Corresponding Author:

Maria Paula de Oliveira Pires

Universidade Federal de São Paulo. Escola Paulista de Enfermagem

Rua Napoleão de Barros, 754

Vila Clementino

CEP: 04024-002, São Paulo, SP, Brasil

E-mail: ma_paulinha@yahoo.com.br
Copyright @ 2015 Revista Latino-Americana de Enfermagem This is an Open Access article distributed under the terms of the Creative Commons Attribution Non-Commercial License (CC BY-NC).

This license lets others distribute, remix, tweak, and build upon your work non-commercially, and although their new works must also acknowledge you and be non-commercial, they don't have to license their derivative works on the same terms. 


\section{Introduction}

In May 2002, at the $55^{\text {th }}$ World Health Assembly, along with the World Health Organization (WHO) and the Member States, the resolution 55.18 was created, which highlights issues related to patient safety, culminating in the creation, in October 2004, of the World Alliance for Patient Safety. The Alliance intend to encourage safe care through good practice assistance and to promote programs aimed at patient safety to be incorporated in health institutions ${ }^{(1)}$.

Among these programs, we highlight the focus on safety in surgical procedures, due to the high number of errors and adverse events. To prevent such damage, the WHO established in 2008, a manual containing a checklist for safe surgery, called Surgical Safety Checklist ${ }^{(2)}$. After the worldwide release, several studies evaluated the effectiveness of the material, demonstrating reduction of perioperative mortality and postoperative complications ${ }^{(3-4)}$. The study evaluated 7.688 patients before and after use of the checklist and confirmed $36.0 \%$ of reduction in serious complications and $47.0 \%$ in mortality ${ }^{(3)}$.

In 2013, the Brazilian Ministry of Health established the National Program of Patient Safety, aiming to encourage safe care in health institutions through the use of protocols, containing good practical assistance focused on patient safety. Among them is the safe surgery protocol(5).

These instruments are directed to safety measures in the intraoperative period, however, pre and postoperative periods are also very important for patient safety.

In Pediatrics, data related to the checklists use to intervene and prepare children and family members during health interventions are scarce. The inclusion of the family in providing information and in the preparation of the child for the surgical procedure is relevant and should focus on patient safety and reduction of their anxiety ${ }^{(6)}$.

Studies evaluated the emotional responses of children undergoing surgery and identified a high preoperative anxiety level - an estimative of between 40 and $75 \%-$, mainly related to fear of the unknown in the moments leading up to the surgery, which can result in future behavior change(7-8).

Another WHO campaign, called "Patient to Patient Safety", reveals the importance of patients' participation in the promotion of their own safety, with the assumption that, when patients participate in the process of care, through collaborative practice and patient-centered care, they are able to promote their own safety in the health system ${ }^{(1,9)}$. However, many factors hinder such participation, especially in health systems in which, culturally, the patient autonomy is opposed by the patronizing attitude and resistance of the health care professional in shared decision-making. Especially concerning pediatric patients, autonomy becomes a challenge ${ }^{(10)}$.

To promote the collaborative participation of the child, family and team in the pediatric patient safety, a Pediatric Surgical Safety Checklist (PSSC) was drafted, and had its content and construct validated. The purpose of this material was to promote the double-checking among children and family along with the team, making sure that the preoperative actions, considered relevant for safe surgery, were performed, by identifying the presence or absence of necessary actions to promote patient safety ${ }^{(11)}$.

Therefore, this research aimed to evaluate the practical application of the PSSC on the preoperative period and verify family satisfaction regarding the use of the material.

\section{Material and method}

Exploratory study that aimed to assess the practical application of the PSSC(11), a material previously validated in content and construct to analyze its use by children submitted to surgical interventions and their families.

The place chosen for the investigation was a pediatric surgery unit of a university hospital in the city of São Paulo. In the unit under study, surgeries of several specialties were performed, of small, medium and large scale.

The study was approved by the Research Ethics Committee of the institution (No. 2114/11). Data collection occurred between October 2012 and January 2013. To constitute the sample, we defined as inclusion criteria the hospitalization in the unit for surgical intervention, of preschool children and adolescents, with development and clinical conditions that allowed the material, consenting the research performance and whose parents agreed to participate. Children who were hospitalized in the afternoon or evening were not included, as well as children whose surgeries were canceled or that had the consent of the responsible or their own denied. At the end of the study period the sample had 60 children and 60 family members. 


\section{Pediatric Surgical Safety Checklist}

The Pediatric Surgical Safety Checklist (PSSC) has 12 items related to interventions to be performed on children in the preoperative period, employing children's language and a playful format. It has drawings that can be painted and a space to be marked by the child and the family with a " $X$ ", or even through painting, as the actions are being carried out by the team. The checklist assumes the form of path to be traveled by the child, since hospitalization until forwarding to the surgical center, contemplating the preoperative period(11).

The PSSC was playfully named "The Path to My Surgery." The 12 items contained in the checklist are: my name is, I arrived at the hospital (date: ______ I I won a bracelet with my name, the nurse explained what will happen to me here at the hospital, the nurse asked me if I had allergies, the nurse told me that I cannot eat or drink anything, today is the day of my surgery, the nurse asked me if I had milk teeth, I was asked to remove earrings/rings/piercing/accessories, I took a shower, my doctor showed me and my family where the surgery will be performed and bye! I'm going in for surgery soon ${ }^{(11)}$.

\section{Practical application of the PSSC}

For the data collection, three instruments were developed with the purpose to contemplate the survey variables, one related to the demographic characteristics of the child and the family, another to the actions related to the PSSC, and the third on the family satisfaction regarding the use of the checklist.

The variables related to the demographic characterization of the sample included gender, age, education, companion's degree of kinship and previous hospitalizations. Of the 12 items that constitute PSSC, 11 were included in the analysis, because the last (Bye! I'm going in for surgery soon) referred to finishing the checklist and does not cover actions directed to promote child safety in the preoperative period. For the analysis, we used the categories: filled item, unfilled item and item not executed by the team. Situations in which we identified that the item had not been filled, we questioned the motive.

Regarding the satisfaction of the family, the variables were categorized using the Likert scale and included: overall satisfaction with the use of the checklist, satisfaction by allowing child care supervision, perception about the child's satisfaction, perception about the child's anxiety reduction, clarity of information, previous use of similar materials presented in the study, ease understanding and filling of the checklist.

The application of the aforementioned instruments was carried out by a researcher, through the presentation of the study and instrumentation of children and family for use of the PSSC at the time of the admission or in a period closer to the surgery. Preceding the data collection, the team was instructed about the checklist use and a pre-test was conducted to evaluate the instruments. After the necessary adjustments, the data collection began.

In the moment of the child's hospitalization in the unit under study, after the presentation of the research to the child and his/her family, and signing of the free and informed consent form, the child and their companions received the PSSC to fill and a set of color pencils to paint the drawings. They were also oriented regarding the return of the checklist.

Preceding the trip to the operating room, about an hour before, the PSSC was collected and an interview was conducted with the relative, which verified the checklist conclusion and satisfaction.

The data obtained were stored in an electronic database, tabulated in spreadsheets with Microsoft Excel ${ }^{\circledR}$ and subsequently analyzed. Descriptive analysis of the data was conducted, being computed the quantitative variables by average, Standard Deviation (SD), median and quartiles, and the qualitative variables, the absolute ( $n$ ) and relative (\%) frequency. For the association analysis, we used the Fisher's exact test and the two sample t-test, through the Minitab ${ }^{\circledR}$ program, version 16.1.

We adopted $5 \%$ of significance level and values of descriptive levels equal to or less than this value $(p \leq 0.05)$ were considered statistically significant.

\section{Results}

The results related to demographic characterization of children and family are presented in Table 1 . The children were mostly male, and age ranged from 3 to 16 years, with an average of 7.5 years. More than half of the children were in elementary school. As for the family, almost all were female and the majority of them were mothers, age ranged from 20 to 64 years, with median of 37 years, and the most common education was high school.

Table 2 shows the applicability of the material. Of the 660 total possible fills related to the 11 items from the checklist, we identified that approximately two-thirds 
were filled $(65.3 \%)$. In the questioning of not filling $(4.7 \%)$, children and family reported that there was lack of time to fill, lack of interest or did not remember the reason. We found that, the team did not execute almost a third of the material items (30.0\%).

The correlations between the filling of the checklist actions and the characteristics of children and family are presented in Tables 3 and 4. We deleted the items in which the reasons justified by the children and family were related to the non-execution of the activity by the team and those who did not present significant justifications, resulting in the inferential analysis of five items.

Table 3 shows that there was no statistical significant difference between the variables gender and children's education, surgery performance, education and kinship of the family member ( $p>0.05)$, analyzing the filling of five items.

Table 4 shows the comparison of the children's and family average age and who did or did not fill the items contained in the checklist. Only the item "I was asked to remove earrings/rings/piercings/accessories", associated with the age of the child, was statistically significant $(p \leq 0.05)$, demonstrating that older children filled more this item than the younger ones.

Regarding the use of material similar to the one presented in this study, only one $(1.7 \%)$ relative mentioned having previous contact. All the family members considered that the PSSC is a material that is easily understandable and almost all of them (98.3\%) reported having no trouble filling it.

The satisfaction study regarding the use of the PSSC is demonstrated in Table 5 . We verified that the majority $(63.3 \%)$ of respondents assessed, in general, the checklist as very good. A significant proportion considered very good (53.3\%) and good (45.0\%) to supervise the care of the child through the use of the material, and highlighted child's satisfaction as very good $(50.0 \%)$ or good $(45.0 \%)$. The majority $(83.3 \%)$ of respondents reported clarity on information and reduction of the child's anxiety.

Table 1 - Demographic characterization of children and their family $(\mathrm{N}=60)$. São Paulo, state of São Paulo, Brazil, 2013

\begin{tabular}{|c|c|c|c|}
\hline Variables related to children & $n(\%)$ & Variables related to family & $n(\%)$ \\
\hline Gender & & Gender & \\
\hline Male & $43(71.7)$ & Female & $58(96.7)$ \\
\hline Female & $17(28.3)$ & Male & $2(3.3)$ \\
\hline Age (years) & & Age (years) & \\
\hline Median $(Q 1-Q 3)^{*}$ & $7.5(4-10)$ & Median (Q1-Q3)* & $37(33-43)$ \\
\hline Min-Max & 3-16 & Min-Max ${ }^{\dagger}$ & 20-64 \\
\hline Education & & Education & \\
\hline Elementary school & $35(58.3)$ & High school & $28(46.7)$ \\
\hline Preschool & $16(26.7)$ & Elementary school & $23(38.3)$ \\
\hline High school & $3(5.0)$ & College & $9(15.0)$ \\
\hline Does not study & $6(10.0)$ & Degree of kinship & \\
\hline Previous hospitalizations & & Mom & $53(88.3)$ \\
\hline No & $44(73.3)$ & Grandparents and great-grandparents & $6(10.0)$ \\
\hline Yes & $16(26.7)$ & Father & $1(1.7)$ \\
\hline
\end{tabular}

* $1^{\text {st }}$ Quartile $-3^{\text {rd }}$ Quartile

+ Minimum - Maximum Age

Table 2 - Checklist actions, according to filling or not by the children or family members, or non-execution of the action by the health team $(\mathrm{N}=60)$. São Paulo, state of São Paulo, Brazil, 2013

\begin{tabular}{|c|c|c|c|}
\hline \multirow{2}{*}{ Checklist items } & Filled & Not filled & Not executed \\
\hline & $\mathbf{N}(\%)$ & $\mathbf{N}(\%)$ & $\mathbf{N}(\%)$ \\
\hline My name is & $59(98.3)$ & $1(1.7)$ & - \\
\hline I arrived at the hospital & $58(96.7)$ & $2(3.3)$ & - \\
\hline Today is the day of my surgery & $55(91,7)$ & $5(8,3)$ & - \\
\hline I won a bracelet with my name & $52(86.7)$ & $4(6.7)$ & $4(6.6)$ \\
\hline The nurse explained what will happen to me here at the hospital & $48(80.0)$ & $2(3.3)$ & $10(16.7)$ \\
\hline The nurse asked me if I had allergies & $48(80.0)$ & $5(8.3)$ & $7(11.7)$ \\
\hline The nurse told me that I cannot eat or drink anything & $47(78.3)$ & $4(6.7)$ & $9(15.0)$ \\
\hline I took a shower & $26(43,3)$ & $1(1.7)$ & $33(55.0)$ \\
\hline The nurse asked me if I have milk teeth & $14(23.3)$ & $1(1.7)$ & $45(75.0)$ \\
\hline I was asked to remove earrings/rings/piercing/accessories & $13(21.7)$ & $4(6.7)$ & $43(71.6)$ \\
\hline My doctor showed me and my family where the surgery will be performed & $11(18,4)$ & $2(3.3)$ & $47(78.3)$ \\
\hline Total & $431(65.3)$ & $31(4.7)$ & $198(30.0)$ \\
\hline
\end{tabular}


Table 3 - Association between the filling of the checklist items and characteristics of the child and family member. São Paulo, state of São Paulo, Brazil, 2013

\begin{tabular}{|c|c|c|c|c|c|c|c|c|c|c|c|c|}
\hline \multirow[t]{2}{*}{ Items* } & \multicolumn{2}{|c|}{$\begin{array}{c}\text { Gender } \\
\text { of the child } n(\%)\end{array}$} & \multicolumn{2}{|c|}{$\begin{array}{l}\text { Education of the } \\
\text { child } n(\%)\end{array}$} & \multicolumn{2}{|c|}{$\begin{array}{c}\text { Previous } \\
\text { hospitalizations n } \\
(\%)\end{array}$} & \multicolumn{2}{|c|}{$\begin{array}{l}\text { Realization of } \\
\text { surgery } n(\%)\end{array}$} & \multicolumn{2}{|c|}{$\begin{array}{c}\text { Education of the } \\
\text { family member } \\
\text { n (\%) }\end{array}$} & \multicolumn{2}{|c|}{$\begin{array}{c}\text { Degree of kinship } \\
\text { of the family } \\
\text { member } n(\%)\end{array}$} \\
\hline & Female & Male & $\begin{array}{c}\mathrm{NE}+ \\
\mathrm{PE}^{\dagger}\end{array}$ & $\mathrm{EF}+\mathrm{EM}^{\ddagger}$ & Yes & No & $\begin{array}{l}\text { On the } \\
\text { day }\end{array}$ & Next day & $\mathrm{EF}^{\S}$ & $E M+S \|$ & Mom & Other \\
\hline \multicolumn{13}{|l|}{$1(n=56)$} \\
\hline Yes & $16(100.0)$ & $36(90.0)$ & 17 (89.5) & $35(94.5)$ & $15(100.0)$ & $37(90.2)$ & $38(90.5)$ & $14(100.0)$ & $20(95.2)$ & $32(91.5)$ & $46(93.8)$ & $6(85.7)$ \\
\hline No & - & $4(10.0)$ & $2(10.5)$ & $2(5.5)$ & - & $4(9.8)$ & $4(9.5)$ & - & $1(4.8)$ & $3(8.5)$ & $3(6.2)$ & $1(14.3)$ \\
\hline$p^{\pi}$ & \multicolumn{2}{|c|}{0.314} & \multicolumn{2}{|c|}{0.598} & \multicolumn{2}{|c|}{0.564} & \multicolumn{2}{|c|}{0.562} & \multicolumn{2}{|l|}{1.000} & \multicolumn{2}{|c|}{0.423} \\
\hline \multicolumn{13}{|l|}{$2(n=53)$} \\
\hline Yes & $14(93.3)$ & 34 (89.5) & $18(94.7)$ & $30(88.2)$ & $12(92.3)$ & $36(90.0)$ & $36(92.3)$ & $12(85.7)$ & $17(85.0)$ & $31(94.0)$ & 43 (91.5) & $5(83.3)$ \\
\hline No & $1(6.7)$ & $4(10.5)$ & $1(5.3)$ & $4(1.8)$ & $1(7.7)$ & $4(100.0)$ & $3(7.7)$ & $2(14.3)$ & $3(15.0)$ & $2(6.0)$ & $4(8.5)$ & $1(16.7)$ \\
\hline$p^{\pi}$ & \multicolumn{2}{|c|}{1.000} & \multicolumn{2}{|c|}{0.643} & \multicolumn{2}{|c|}{1.000} & \multicolumn{2}{|c|}{0.598} & \multicolumn{2}{|l|}{0.353} & \multicolumn{2}{|c|}{0.465} \\
\hline \multicolumn{13}{|c|}{$3(n=51)$} \\
\hline Yes & $12(92.3)$ & $35(92.1)$ & $16(88.8)$ & $31(94.0)$ & $13(100.0)$ & 34 (89.5) & $37(92.5)$ & $10(91.0)$ & $16(88.8)$ & $31(94.0)$ & $42(91.3)$ & $5(100.0)$ \\
\hline No & $1(7.7)$ & $3(7.9)$ & $2(11.2)$ & $2(6.0)$ & - & $4(10.5)$ & $3(7.5)$ & $1(9.0)$ & $2(11.2)$ & $2(6.0)$ & $4(8.7)$ & - \\
\hline$p^{\pi}$ & \multicolumn{2}{|c|}{1.000} & \multicolumn{2}{|c|}{0.607} & \multicolumn{2}{|c|}{0.561} & \multicolumn{2}{|c|}{1.000} & 0.607 & & \multicolumn{2}{|c|}{1.000} \\
\hline \multicolumn{13}{|c|}{$4(n=60)$} \\
\hline Yes & $15(88.2)$ & $40(93.0)$ & $19(86.3)$ & $36(94.7)$ & $15(93.7)$ & $40(91.0)$ & $42(91.3)$ & $13(92.8)$ & 21 (91.3) & $34(91.8)$ & $48(90.5)$ & $7(100.0)$ \\
\hline No & $2(11.8)$ & $3(7.0)$ & $3(13.7)$ & $1(5.3)$ & $1(6.3)$ & $4(9.0)$ & $4(8.7)$ & $1(7.2)$ & $2(8.7)$ & $3(8.2)$ & $5(9.5)$ & - \\
\hline$p^{\pi}$ & \multicolumn{2}{|c|}{0.615} & \multicolumn{2}{|c|}{0.345} & \multicolumn{2}{|c|}{1.000} & \multicolumn{2}{|c|}{1.000} & 1.000 & & 1.0 & 00 \\
\hline $5(n=17)$ & & & & & & & & & & & & \\
\hline Yes & $6(85.7)$ & $7(70.0)$ & $2(50.0)$ & $11(84.6)$ & $2(66.7)$ & 11 (78.5) & $8(80.0)$ & $5(71.5)$ & $4(66.6)$ & $9(81.8)$ & $12(80.0)$ & $1(50.0)$ \\
\hline No & $1(14.3)$ & $3(30.0)$ & $2(50.0)$ & $2(15.4)$ & $1(33.3)$ & $3(21.5)$ & $2(20.0)$ & $2(28.5)$ & $1(33.4)$ & $2(18.2)$ & $3(20.0)$ & $1(50.0)$ \\
\hline$p^{\pi}$ & 0.6 & & 0.2 & 218 & 1.0 & & & 000 & 0.584 & & 0.4 & 26 \\
\hline
\end{tabular}

* Checklist items: 1. I won a bracelet with my name; 2 . The nurse asked me if I had allergies; 3 . The nurse told me that I cannot eat or drink anything; 4. Today is the day of my surgery; 5 . I was asked to remove earrings/rings/piercings/accessories

+ Does not study and preschool

₹ Elementary and high school

$\S$ Elementary school

II High school and College

If Fisher's exact test

Table 4 - Association between the filling of checklist items and the children's and family members' age ( $N=60)$ São Paulo, state of São Paulo, Brazil, 2013

\begin{tabular}{|c|c|c|c|c|c|c|}
\hline \multirow{2}{*}{ Checklist items } & \multicolumn{3}{|c|}{ Age of the child (years) } & \multicolumn{3}{|c|}{ Age of the family member (years) } \\
\hline & $\mathbf{P}^{*}$ & $\mathbf{N P}^{\dagger}$ & $\mathbf{p}^{\ddagger}$ & $\mathbf{P}^{*}$ & $\mathrm{NP}^{\dagger}$ & $\mathbf{p}^{\ddagger}$ \\
\hline I won a bracelet with my name $(n=56)$ & $7.8(3.3)$ & $6.2(3.2)$ & 0.399 & $37.9(9.0)$ & $41.3(16.0)$ & 0.707 \\
\hline The nurse asked me if I had allergies $(n=53)$ & $7.4(3.4)$ & $9.0(2.7)$ & 0.296 & $37.5(8.9)$ & $36.4(10.3)$ & 0.817 \\
\hline The nurse told me that I cannot eat or drink anything $(n=51)$ & $7.4(3.0)$ & $7.5(3.7)$ & 0.996 & $37.0(9.1)$ & $33.7(6.1)$ & 0.387 \\
\hline Today is the day of my surgery $(n=60)$ & $7.6(0.4)$ & $8.2(4.9)$ & 0.810 & $38.1(9.5)$ & $38.6(12.3)$ & 0.937 \\
\hline I was asked to remove earrings/rings/piercing/accessories & $10.6(3.2)$ & $6.5(1.7)$ & 0.008 & $36.3(6.6)$ & $38.3(13.6)$ & 0.809 \\
\hline
\end{tabular}

* Filled (average \pm standard deviation)

+ Not filled (average \pm standard deviation)

\# Two-sample t-test 
Table 5 - Family satisfaction regarding the use of the checklist $(N=60)$. São Paulo, state of São Paulo, Brazil, 2013

\begin{tabular}{lc}
\hline \multicolumn{1}{c}{ Satisfaction } & N (\%) \\
\hline Global with the checklist & $38(63.3)$ \\
Very good & $22(36.7)$ \\
Good & - \\
Neither good, nor bad & - \\
Bad & - \\
Terrible & \\
The child's & $30(50.0)$ \\
Very good & $27(45.0)$ \\
Good & $3(5.0)$ \\
Neither good, nor bad & - \\
Bad & - \\
Terrible & \\
To supervise the child's care & $32(53.3)$ \\
Very good & $27(45.0)$ \\
Good & $1(1.7)$ \\
Neither good, nor bad & - \\
Bad & \\
Terrible & \\
Reduction of the child's anxiety & - \\
Reduced & \\
Sometimes reduced & \\
I don't know & \\
Did not reduce & \\
Clarity of information & \\
Clear & $50(83.3)$ \\
Some clear some unclear & $5(8.3)$ \\
Unclear & \\
I don't know & - \\
\hline & \\
\hline
\end{tabular}

\section{Discussion}

The strategy used to conduct the preoperative actions with the purpose of reducing risks to the child was the duty of the PSSC, application form in folder format ${ }^{(11-12)}$. This type of material showed high acceptance among children and family members with the checklist, supporting the proposal to participate, by checking each item, after being completed by the team. However, when not filled by the children or their relative, this fact could be related to non-execution of the action by the team. In such situations, the children and family members did not ask the team to perform the activities. There was only one family member who asked the team and required the act, in this case the realization of the bath, because he understood that there was a need for this care in the preoperative period, as mentioned in the checklist.
During the presentation of the PSSC to the family and children, no reference was made to ask the team to perform the activities contained in the checklist, because it is not the aim of the study. Several factors can influence the non-execution of some items by the team, such as the small number of professionals, the overload of activities, the high number of patients, as well as the lack of interaction with the children and their family.

The use of the checklist can facilitate this interaction, because, as the patient and the family are oriented about what should happen in the preoperative period, and have a place to record these actions, as occurs with the PSSC, there is the possibility of shared decision making with the team.

A review study, carried out in 2014, found that children who were oriented about the preoperative period achieved reduction in anxiety level. However, this reduction was related mainly to children over 4 years of age ${ }^{(13)}$. Yet, it is recommended that the preparation should occur for children of any age, since it can be accomplished by different methods, increasing the knowledge of the situation experienced by the child and family, and promoting distraction ${ }^{(14)}$.

Though the anxiety level was not evaluated in this research, through specific scales, we asked about the family's perception regarding it. Most of them reported that the children did not demonstrate such feeling.

These results corroborate those of the research conducted in three hospitals in Lisbon, in 2013, which showed that the supply of preoperative materials to children of 8 to 12 years of age, containing educational information, reduced their concerns when compared to those who had no preparation ${ }^{(15)}$.

We should also highlight that the relatives that are oriented on this period also exhibit lower levels of anxiety ${ }^{(13)}$. The insertion of the family members in the preoperative care, not only satisfies them, but also generates feelings of security and commitment in the process. A research conducted in Dublin, with the participation of parents during the checklist application, proposed by $\mathrm{WHO}$, at the moment before the anesthetic induction, found that there was no prejudice in the development of surgery, and the anxiety levels of the family members was reduced, since 97\% reported feeling reassured by being close to the children. The study suggested that parents should always be involved in the care of children and that professionals considered this process as mandatory, since it is beneficial for the team, child and family ${ }^{(16)}$. A culture of safety needs, therefore, to evolve this care along with the team ${ }^{(6)}$. 
When analyzing the PSSC, there were items that were not carried out by the team for more than $50 \%$ of the children and that could bring security risks to them. Among these were milk teeth identification, removal of accessories, preoperative bath and laterality of surgery.

Children the age of those that composed the sample of the study were in the process of exchanging their teeth, being extremely important to check them to prevent their detachment at the time of intratracheal intubation, for patients who are undergoing general anesthesia(17).

Regarding the removal of accessories, it is worth mentioning that often pre-teens and teenagers use these, so guidance for the removal is needed, as it prevents the occurrence of burns during the electric scalpel use, finger tourniquet application and possibility of loss $^{(18)}$.

Another relevant item is the execution of the preoperative bath to prevent surgical site infections, considered the main sources of morbidity and mortality among patients undergoing surgery, increasing the length of stay and the cost of the procedure ${ }^{(19)}$. According to institutional protocol, the shower should occur six hours before surgery with use of germicide, however, in elective surgeries, the children usually showered at their residence. However, the interval between the bath and the surgery exceeded the recommended and the team did not question the bath time, only if it had been done.

As to laterality, the local to be operated has to be delimited, especially those with multiple structures, during the preoperative and preanesthetic consultation, this activity is essential for the safe and quality care, including the patient's confirmation, local and correct execution, to avoid possible errors in surgical sites, considering that repeated information helps in the security procedure(2). In a previous study, the lack of participation of the team in this step occurred in $3.6 \%$ of cases ${ }^{(20)}$.

The team has difficulty in using new instruments, as already demonstrated in research related to use the Safety Surgical Checklist ${ }^{(21-22)}$, however, due to the relevance of those in security and reduction of errors and adverse events in health care, through double checking and confirmation of essential tasks to be performed(2), it is expected that, gradually, the professionals, along with the aid of health institutions, seek efforts to promote the commitment of the patient safety culture.

A study in the pediatric area showed that the use of the checklist, suggested by WHO, can improve patient safety. They verified that adhesion to the list enabled detection and prevention of human error, equipment malfunction, and identification of areas that needed reinforcement, reorientation and modernization ${ }^{(20)}$.
That way, the study sought to provide subsidy for the improvement of this practice, offering information not only to the families, but also to the children, as well as preoperative care for their safety, getting participation in the checklist use.

Therefore, we identified that the PSSC can inform and prepare children and family members for surgical procedures, in addition to the guidelines and activities of the team, providing safe and quality care. Everyone involved in the care can assist the child in the process of adapting and improving health(23)

The study has limitations regarding the analysis of the practical applicability of the checklist, due to the size of the sample, the realization in only one center, having no items checked by the child and family because they were not performed by the team and necessity to prove its effect on reduction of adverse events that may compromise patients' safety. However, we highlight the innovative nature and the possibility of expanding the use of the material, validating its usefulness as an instrument that introduces the child and the family to the process of care and has the potential to increase their safety during surgical procedures.

\section{Conclusion}

The application in practice of the Pediatric Surgical Safety Checklist showed that occurred involvement of children and their family members to fill the items. The difference in the filling and the characteristics of children and family members was significant in only one item related to the removal of accessories and age, in which the older ones filled more than the others. We believe that the PSSC can contribute to the systematization of the assistance and double-checking, as long as everyone involved understand the need to perform activities related to the period. We also identified the interest children and their family members took in the checklist, as well as greater family satisfaction and children's anxiety reduction. That way, the material can collaborate to initiate changes related to pediatric patient safety culture, promoting commitment of all those involved in the process, promoting their satisfaction.

\section{References}

1. World Health Organization (WHO). World Alliance for Patient Safety. Forward Programme 2008-2009. Geneve: WHO; 2008. 
2. World Health Organization (WHO). The second global patient safety challenge: safe surgery saves lives. Geneve: WHO; 2009.

3. Haynes AB, Weiser TG, Berry WR, Lipsitz SR, Breizat AH, Dellinger $E P$, et al. Safe surgery saves lives study group. A surgical safety checklist to reduce morbidity and mortality in a global population. N Engl J Med. 2009;360(5):491-9. 4. De Vries EN, Prins HA, Crolla RMPH, Den Outer AJ, Van Andel G, Van Helden $\mathrm{SH}$, et al; SURPASS Collaborative Group. Effect of a comprehensive surgical safety system on patient outcomes. N Engl J Med. 2010;363(20):1928-37. 5. Ministério da Saúde (BR). Portaria No 529, de $1^{\circ}$ de abril de 2013. Institui o Programa Nacional de Segurança do Paciente (PNSP) [Internet]. 2013 [Acesso 14 jun 2014]; Disponível em: http://bvsms.saude.gov.br/bvs/ saudelegis/gm/2013/prt0529_01_04_2013.html.

6. Corbally MT, Tierney E. Parental involvement in the preoperative surgical safety checklist is welcomed by both parents and staff. Int J Pediatr. 2014;2014:791490. 7. Vagnoli L, Caprilli S, Messeri A. Parental presence, clowns or sedative premedication to treat preoperative anxiety in children: what could be the most promising option? Paediatr Anaesth. 2010;20:937-43.

8. Cumino DO, Cagno G, Gonçalves VFZ, Wajman DS, Mathias LAST. Impact of preanesthetic information on anxiety of parents and children. Rev Bras Anestesiol. 2013;63(6):473-82.

9. Waterman AD, Gallagher TH, Garbutt J, Waterman BM, Fraser V, Burroughs TE. Brief report: hospitalized patients' attitudes about and participation in error prevention. J Gen Inter Med. 2006;21:367-70.

10. Henriksen K, Oppenheimer C, Leape LL, Hamilton K, Bates DW, Sheridan S, et al. Envisioning Patient Safety in the Year 2025: Eight Perspectives. In: Henriksen K, Battles JB, Keyes MA, Grady ML. Advances in Patient Safety: New Directions and Alternative Approaches. Rockville (MD): Agency for Healthcare Research and Quality; 2008. [Acesso 14 set 2014]. Disponível em: http://www.ncbi.nlm.nih.gov/books/NBK43618/

11. Pires MPO, Pedreira MLG, Peterlini MAS. Safe pediatric surgery: development and validation of preoperative interventions checklist. Rev. Latino-Am. Enfermagem. 2013;21(5):1080-7.

12. Inott $T$, Kennedy BB. Assessing Learning Styles: practical tips for patient education. Nurs Clin North Am. 2011;46(3):313-20.

13. Copanitsanou $P$, Valkeapää K. Effects of education of pediatric patients undergoing elective surgical procedures on their anxiety - a systematic review. J Clin Nurs. 2014; 23(7-8):940-54.
14. Broering CV, Crepaldi MA. Psychological preparation and stress of children undergoing surgery. Psicol Estud. 2011;16(1):15-23.

15. Fernandes SC, Arriaga P, Esteves F. Providing preoperative information for children undergoing surgery: a randomized study testing different types of educational material to reduce children's preoperative worries. Health Educ Res. 2014; 29(6):1058-76.

16. van Klei WA, Hoff R, van Aarnhem EE, Simmermacher RK, Regli LP, Kappen TH, et al. Effects of the introduction of the WHO "Surgical Safety Checklist" on in-hospital mortality: a cohort study. Ann Surg. 2012;255(1):44-9. 17. Yasny JS. Perioperative dental considerations for the anesthesiologist. Anesth Analg. 2009;108:1564-73. In: Bejeh MIR, Karim P, Arash P. Neglected Orodental Facts during General Anesthesia and Intensive Care Unit Admission in Pediatric Population. Rev Bras Anestesiol. 2012;62(6):849-51.

18. Christoforo BEB, Carvalho DS. Nursing care applied to surgical patient in the pre-surgical period. Rev Esc Enferm USP. 2009;43(1):14-22.

19. Dias GA. Prophylactic antibiotic therapy and/or therapeutic in patients undergoing plastic surgery: a necessity? Rev Bras Cir Plást. 2010;25(3):423-7.

20. Oak SN, Dave NM, Garasia MB, Parelkar SV. Surgical checklist application and its impact on patient safety in pediatric surgery. J Postgrad Med. 2015;61(2):92-4.

21. Fourcade A, Blache JL, Grenier C, Bourgain JL, Minvielle E. Barriers to staff adoption of a surgical safety checklist. BMJ Qual Saf. 2012;21(3):191-7.

22. Cunat C, Flatin V, Viale JP. Stratégie de déploiement de la check-list dans un CHU. Ann Fr Anesth Reanim. 2011;30:484-8.

23. Knoerl AM, Esper KW, Hasenau SM. Cultural Sensitivity in Patient Health Education. Nurs Clin North Am. 2011;46(3):335-40. 